\title{
Associação da implantação de Equipes de Saúde Bucal com ações coletivas e exodontia no Estado do Paraná, Brasil
}

\author{
Association of Oral Health Teams implantation \\ with collective actions and extractions in Paraná State, Brazil
}

Paula Mayumi Siqueira (https://orcid.org/0000-0001-5421-0247) ${ }^{1}$
Josely Emiko Umeda (https://orcid.org/0000-0003-1106-4344) ${ }^{1}$
Raquel Sano Suga Terada (https://orcid.org/0000-0003-1344-9870) ${ }^{1}$
Amanda Fonquete Giozet (https://orcid.org/0000-0002-4387-1733) ${ }^{1}$
Juliana Squizatto Leite (https://orcid.org/0000-0001-7084-7467) ${ }^{2}$
Moacir Paludetto Junior (https://orcid.org/0000-0002-3039-6611) ${ }^{3}$
Cacilda Castelo Branco Lima (https://orcid.org/0000-0002-2977-6035)
Mitsue Fujimaki (https://orcid.org/0000-0002-7824-3868) ${ }^{1}$
${ }^{1}$ Departamento de Odontologia, Universidade Estadual de Maringá. Av. Mandacaru 1550, Parque das Laranjeiras. 87083240 Maringá PR Brasil. mayumi.siqueira2312@ gmail.com

${ }^{2}$ Universidade Estadual de Ponta Grossa. Ponta Grossa PR Brasil.

${ }^{3}$ Secretaria Municipal de Saúde de Arapongas. Arapongas PR Brasil.

${ }^{4}$ Departamento de Patologia e Clínica Odontológica, Universidade Federal do Piauí. Teresina PI Brasil.

\begin{abstract}
This article aims to monitor the indicators of outpatient production of Dentistry and to evaluate the association of collective action and extraction indicators with the number of Oral Health Teams (OHT) between 2006 and 2015 in Paraná. In this longitudinal ecological study, consolidated secondary data were analyzed (collective actions of supervised brushing-SB, topical application of fluoride-TAF, fluoride mouthwash-FM, oral examination for epidemiological purposes-OE and extractions of permanent teethEX) from the Ambulatory Information System (SIA-SUS) and OHT numbers from the National Registry System of Health Establishments. Descriptive analyzes and Pearson's correlation were performed, with significance level of $p<0.05$. It was verified the increase of the OHT implantation over time and a strong positive correlation with collective procedures of $S B(r=0.78 ; p=0.007)$ and FM ( $r=0.76 ; p=0.011)$ and moderate negative correlation with EX ( $r=-0.53)$. It was concluded that the evaluated indicators showed that the implementation of Oral Health Teams may have contributed to changes to the healthcare model, with an increase in preventive collective procedures and reduction of tooth loss in Paraná.

Key words Preventive Dentistry, Health Status Indicators, Public Health
\end{abstract}

Resumo $O$ artigo tem por objetivo monitorar a produção ambulatorial em Odontologia e avaliar a associação de indicadores de ação coletiva e exodontia com o número de Equipes de Saúde Bucal (ESB) entre 2006 e 2015, no Paraná. Neste estudo ecológico longitudinal foram analisados dados secundários consolidados (ações coletivas de escovação supervisionada-ES, aplicação tópica de flúor-ATF, bochecho fluorado-BF, exame bucal com finalidade epidemiológica-EB e exodontia de dente permanente-EXO) do Sistema de Informação Ambulatorial e números de ESB no Sistema de Cadastro Nacional de Estabelecimentos de Saúde. Foram realizadas análises descritivas e correlação de Pearson, com nível de significância de $p<0,05$. Verificou-se um aumento de ESB implantadas ao longo do tempo e correlação forte positiva com procedimentos coletivos de ES $(r=0,78 ; p=0,007)$ e BF $(r=0,76 ; p=0,011)$ e correlação moderada negativa com EXO ( $r=-0,53)$. Conclui-se que os indicadores avaliados demonstraram que a implantação de Equipes de Saúde Bucal pode ter contribuído para mudanças no modelo de atenção, com aumento dos procedimentos coletivos preventivos $e$ redução de perdas dentárias no Paraná.

Palavras-chave Odontologia Preventiva, Indicadores Básicos de Saúde, Saúde Pública 


\section{Introdução}

O conhecimento da população e sua condição bucal são fundamentais para o desenvolvimento de propostas de ações adequadas às necessidades de diferentes grupos populacionais. A importância de avaliar a aplicação dos recursos e seu impacto na saúde da população faz com que sejam elaboradas estratégias e normativas específicas para monitorar a qualidade e o efeito dos atendimentos na atenção básica ${ }^{1}$. O planejamento deve privilegiar ações de prevenção e promoção da saúde, sendo de grande valia a realização do monitoramento e avaliação das mesmas. No entanto, o monitoramento dos indicadores de produção ambulatorial em Odontologia associado à implantação de Equipes de Saúde Bucal no estado do Paraná ainda não foi amplamente estudado e divulgado.

Para tanto, os sistemas de informação em saúde são ferramentas disponíveis para auxiliar no planejamento para a melhoria dos índices epidemiológicos das doenças. No Sistema Único de Saúde (SUS), o Sistema de Informações Ambulatoriais (SIA) e o Sistema de Informação da Atenção Básica $(\mathrm{SIAB})^{2}$, que vêm sendo substituídos gradativamente pelo e-SUS 3 , são instrumentos de gestão importantes que possibilitam o monitoramento e avaliação dos serviços e auxiliam na tomada de decisões. Assim, a utilização dos dados dos sistemas de informações disponíveis torna-se necessária para o planejamento adequado das ações em saúde ${ }^{4-8}$, integrado à realidade orçamentária, visando a melhoria dos serviços?.

O Pacto de Indicadores da Atenção Básica é um instrumento de negociação entre os gestores das esferas municipal, estadual e federal e estabelece as metas anuais a serem alcançadas conforme os indicadores previamente acordados, com o objetivo de melhoria da atenção básica e da saúde da população ${ }^{10}$. No estudo de Teixeira et al. ${ }^{11}$, foi avaliado o número de procedimentos odontológicos realizados em um município do Rio Grande do Sul, no período de 2000 a 2007 utilizando os dados do Ministério da Saúde e foi observado um aumento no número de procedimentos individuais básicos e de primeira consulta, com redução relativa à proporção de exodontias, resultado da expansão dos serviços odontológicos de acordo com a livre demanda ${ }^{10}$. Em outro estudo realizado por Baldani et al..$^{12}$, as condições socioeconômicas foram relacionadas com a provisão de serviços odontológicos nas 399 cidades do Paraná de 1998 a 2005, tendo como resultado a expansão dos serviços odontológicos, assim como, maior provisão de recursos para municípios com piores indicadores socioeconômicos, demonstrando a importância de monitorar os dados do SIA-SUS para reorganizar a oferta de serviços de saúde conforme a necessidade local. No entanto, poucos trabalhos ${ }^{5,13-16}$ na literatura têm demonstrado as mudanças ocorridas na produção de atividades coletivas e na redução de exodontias após a implantação das Equipes de Saúde Bucal (ESB) na Estratégia Saúde da Família.

Deste modo, o objetivo deste estudo foi realizar o monitoramento de indicadores de produção ambulatorial em Odontologia e avaliar a associação dos indicadores de ação coletiva e exodontia com o número de Equipes de Saúde Bucal implantadas no período de 2006 a 2015 no estado do Paraná.

\section{Materiais e métodos}

Trata-se de um estudo ecológico longitudinal, com uso de dados secundários de domínio público, consolidados entre 2006 e 2015, nos 399 municípios do Paraná, Brasil. Foram utilizadas informações relativas ao acesso e aos serviços de atenção básica à saúde bucal, caracterizadas pelos indicadores. A fase de coleta de dados foi obtida por meio de consultas no sítio do Departamento de Informática do SUS (DATASUS) do Ministério da Saúde dos seguintes dados:

- Sistema de Informações Ambulatoriais do SUS (SIA-SUS): Ação Coletiva de Bochecho Fluoretado (BF) - código 03011038 (até 2007) e código 0101020023 (a partir de 2008); Ação Coletiva de Escovação Dental Supervisionada (ES) código 03011020 (até 2007) e código 0101020031 (a partir de 2008); Aplicação Tópica de Flúor (ATF) - código 03011046 (até 2007) e código 0101020015 (a partir de 2008); Ação Coletiva de Exame Bucal com Finalidade Epidemiológica (EB) - código 03011058 (até 2007) e código 0101020040 (a partir de 2008) e Exodontia de Dentes Permanentes (EXO) - código 03041026 (até 2007) e o código 0414020138 (a partir de 2008);

- Cadastro Nacional de Estabelecimentos de Saúde (CNES): equipes de saúde bucal implantadas por ano;

- Instituto Brasileiro de Geografia e Estatística $(\mathrm{IBGE})^{17}$ : população estimada do estado do Paraná por ano.

Os municípios que não apresentaram nenhuma informação foram excluídos. Após a obtenção dos dados secundários, os indicadores de 
produção ambulatorial em Odontologia foram calculados utilizando a fórmula abaixo:

$$
\begin{gathered}
\text { Indicador anual de produção }= \\
1.000 \times \text { Quantidade de } \\
\text { procedimentos realizados no ano } \\
\text { População estimada no ano }
\end{gathered}
$$

A cobertura das Equipes de Saúde Bucal (ESB) foi calculada utilizando a fórmula abaixo ${ }^{18}$.

Cobertura da ESB $=$

Número de ESB x 3.000 x 100

População estimada no ano

\section{Análise Estatística}

O processamento dos dados e análise estatística foram realizados no programa Statistical Package for the Social Sciences (SPSS ${ }^{\circledR}$ para Windows, versão 20.0, Armonk, NY, EUA: IBM Corp). Foi realizada a estatística descritiva de todas as variáveis. O teste Shapiro-Wilk foi usado para avaliar a distribuição de normalidade das variáveis. As variáveis apresentaram distribuição normal ( $p>0,05)$ : BF $(0,734)$, ES $(0,589)$, ATF $(0,372)$, EB (0,003), EXO $(0,588)$.

Para verificar as associações entre as médias dos anos dos indicadores dos procedimentos ambulatoriais (BF, ES, ATF, EB, EXO) e a cobertura de equipes de saúde bucal foi realizada a correlação de Pearson. O nível de significância estatística adotado foi de $\mathrm{p}<0,05$.

O nível de similaridade entre as variáveis ESB, EXO, ATF, BF e ES foi analisado com a técnica de análise multivariada pelo método de agrupamento de variáveis (Cluster). Além disso, foi utilizada a técnica de análise multivariada pelo método de componentes principais para complementar as informações obtidas pelo método de Cluster.

\section{Resultados}

Os valores dos indicadores referentes à BF, ES, EB, ATF e EXO estão apresentados na Tabela 1. Foi possível observar aumento em todos os indicadores de procedimentos ambulatoriais (BF aumento de $57,15 \%$, ES aumento de $98,36 \%$, ATF aumento de 30,98\%, EB aumento de 108,11\%), exceto para exodontia (redução de 37,09\%). No decorrer dos anos houve um declínio nos procedimentos de EXO, principalmente após 2011. Na avaliação deste indicador tem-se que em 2006 o indicador de EXO era de 43,86 e em 2015 reduziu para 27,59 (Tabela 1). A hipótese de uma possível interferência do aumento populacional sobre a redução de EXO não pode ser considerada, já que os demais procedimentos ambulatoriais (BF, ES, ATF e EB) foram aumentados, tendo sido utilizado o mesmo denominador populacional para os cálculos dos indicadores, de acordo com os respectivos anos compreendidos no estudo. A população do estado do Paraná não apresentou picos discrepantes de crescimento, sendo representada por aumentos gradativos, assegurando que os valores obtidos dos indicadores não sofreram interferências desta variável.

$\mathrm{O}$ número de ESB apresentou um aumento gradativo no período entre 2006 e 2015 no estado do Paraná, alcançando 1.286 equipes em 2015 (Gráfico 1). O aumento no número de ESB melhorou a cobertura da população do estado, partindo da situação de $25,33 \%$ de cobertura populacional em 2006, para 34,56\% em 2015.

Por meio da Correlação de Pearson, foi possível estabelecer uma correlação entre os indicadores dos procedimentos coletivos e a exodontia com o indicador das equipes de saúde bucal, conforme demonstrado no Quadro 1. Houve uma correlação negativa entre EXO e BF ( $r=-$ 0,61), entre EXO e ESB implantadas $(r=-0,53)$; e entre EXO e ES ( $r=-0,43)$. Por outro lado, houve forte correlação positiva e significativa entre ESB com os procedimentos coletivos de ES $(r=0,78$;

Tabela 1. Série histórica dos indicadores dos procedimentos de bochechos fluorados (BF), escovação supervisionada (ES), aplicação tópica de flúor (ATF), exame bucal com finalidade epidemiológica (EB) e exodontia de dentes permanentes (EXO) no período de 2006 a 2015, no estado do Paraná.

\begin{tabular}{lccccc}
\hline \multirow{2}{*}{ Ano } & \multicolumn{5}{c}{ Indicadores } \\
\cline { 2 - 6 } & BF & ES & ATF & EB & EXO \\
\hline 2006 & 393,56 & 219,46 & 21,51 & 9,31 & 43,86 \\
2007 & 466,76 & 376,46 & 28,30 & 12,66 & 42,14 \\
2008 & 416,29 & 378,32 & 36,80 & 15,92 & 55,71 \\
2009 & 365,64 & 348,15 & 32,88 & 18,69 & 60,47 \\
2010 & 458,07 & 395,12 & 37,73 & 17,26 & 46,62 \\
2011 & 608,92 & 508,61 & 35,96 & 55,23 & 50,90 \\
2012 & 532,37 & 463,92 & 29,54 & 13,12 & 47,06 \\
2013 & 515,68 & 415,13 & 26,67 & 31,59 & 33,16 \\
2014 & 548,07 & 655,19 & 35,97 & 19,40 & 27,62 \\
2015 & 618,48 & 435,33 & 28,16 & 19,38 & 27,59 \\
\hline
\end{tabular}

Fonte: Elaborado pelos autores. Consulta na base de dados do Sistema de Informações Ambulatoriais do SUS (SIA-SUS) e Instituto Brasileiro de Geografia e Estatística (IBGE) para a construção dos indicadores. 


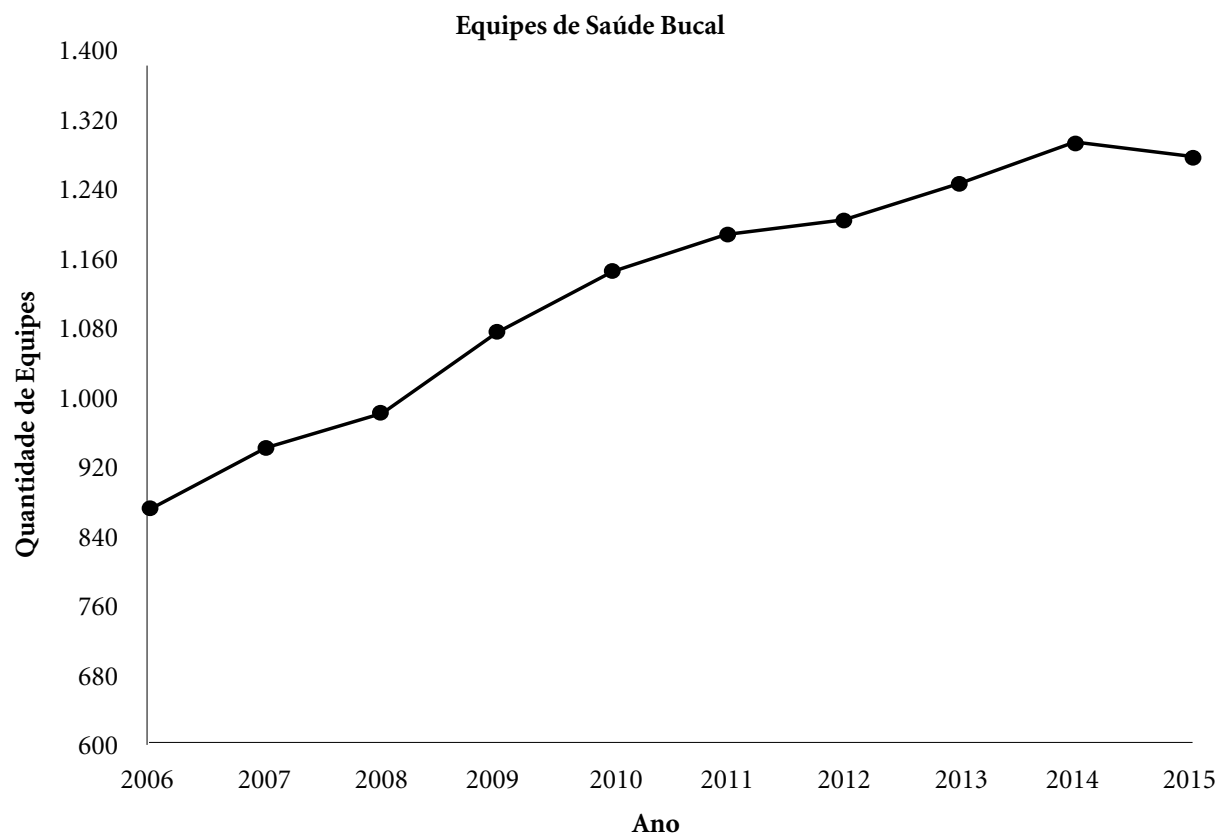

Gráfico 1. Implantação de Equipes de Saúde Bucal no período de 2006 a 2015, no estado do Paraná.

Fonte: Elaborado pelos autores. Consulta no Cadastro Nacional de Estabelecimentos de Saúde (CNES) para a obtenção do número de Equipes de Saúde Bucal (ESB).

Quadro 1. Matriz de correlação de Pearson entre os indicadores dos procedimentos de bochechos fluorados (BF), escovação supervisionada (ES), aplicação tópica de flúor (ATF), exame bucal com finalidade epidemiológica (EB) e exodontia de dentes permanentes (EXO) com o indicador de Equipes de Saúde Bucal (ESB) implantadas no período entre 2006 e 2015.

\begin{tabular}{|l|r|r|r|r|r|r|}
\hline Variáveis & $\begin{array}{c}\text { ESB } \\
\text { r }\end{array}$ & $\begin{array}{c}\text { BF } \\
\text { r }\end{array}$ & $\begin{array}{c}\text { ES } \\
\mathbf{r}\end{array}$ & $\begin{array}{c}\text { ATF } \\
\mathbf{r}\end{array}$ & $\begin{array}{c}\text { EB } \\
\mathbf{r}\end{array}$ & $\begin{array}{c}\text { EXO } \\
\mathbf{r}\end{array}$ \\
\hline ESB & 1 & $0,76^{*}$ & $0,78^{*}$ & 0,31 & 0,43 & $-0,53$ \\
\hline BF & $0,76^{*}$ & 1 & $0,69^{*}$ & 0,10 & 0,55 & $-0,61$ \\
\hline ES & $0,78^{*}$ & $0,69^{*}$ & 1 & 0,55 & 0,40 & $-0,43$ \\
\hline ATF & 0,31 & 0,10 & 0,55 & 1 & 0,32 & 0,31 \\
\hline EB & 0,43 & 0,55 & 0,40 & 0,32 & 1 & 0,05 \\
\hline EXO & $-0,53$ & $-0,61$ & $-0,43$ & 0,31 & 0,05 & 1 \\
\hline
\end{tabular}

$\mathrm{r}=$ coeficiente de correlação de Pearson; ${ }^{*} \mathrm{p}<0,05$.

Fonte: Elaborado pelos autores.

$\mathrm{p}=0,007)$ e $\mathrm{BF}(\mathrm{r}=0,76 ; \mathrm{p}=0,011)$, moderada correlação positiva e significativa entre BF e ES $(\mathrm{r}=0,69 ; \mathrm{p}=0,027)$ e baixa correlação entre EXO e $\operatorname{ATF}(\mathrm{r}=0,31)$ (Quadro 1).
$\mathrm{Na}$ análise multivariada pelo método de Cluster, foi possível observar que o procedimento de EXO apresentou maior similaridade com ATF, de $65,46 \%$ em comparação aos demais indicadores, como demonstrado no dendrograma da Figura 1. Os graus de similaridade mais altos foram entre ESB e ES com valor de $89,17 \%$ e ambos têm $84,49 \%$ de similaridade com a BF (Figura 1). Além disso, foi realizada a análise multivariada pelo método da análise de componentes principais, apresentando as correlações positivas e negativas entre as variáveis BF, ES, ESB, ATF e EXO, demonstrada na Gráfico 2. Na análise dos fatores, o determinante da matriz foi 0,030 , a estatística de KMO foi 0,66 e o teste de Bartlett foi 0,011.

\section{Discussão}

Estudos longitudinais em saúde pública são importantes ferramentas para o planejamento de políticas públicas com o objetivo de melhorar a qualidade e o impacto da atenção à saúde da população $^{13}$. Segundo o Ministério da Saúde, dados epidemiológicos e informações territoriais de- 


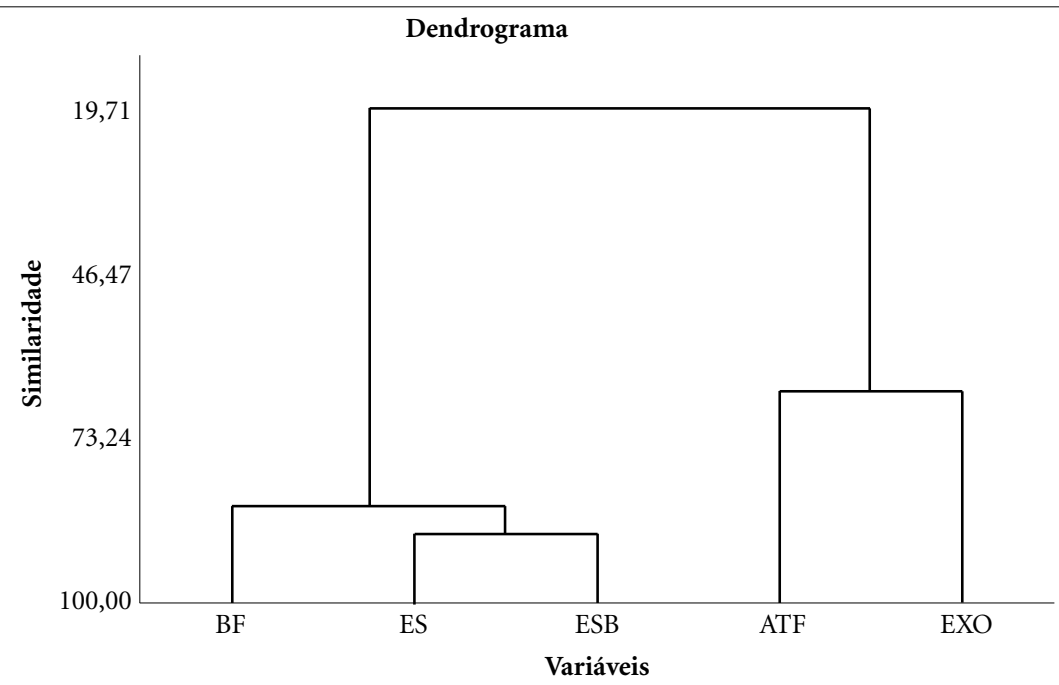

Figura 1. Dendrograma das relações entre as variáveis BF, ES, ESB, ATF e EXO.

Fonte: Elaborado pelos autores.

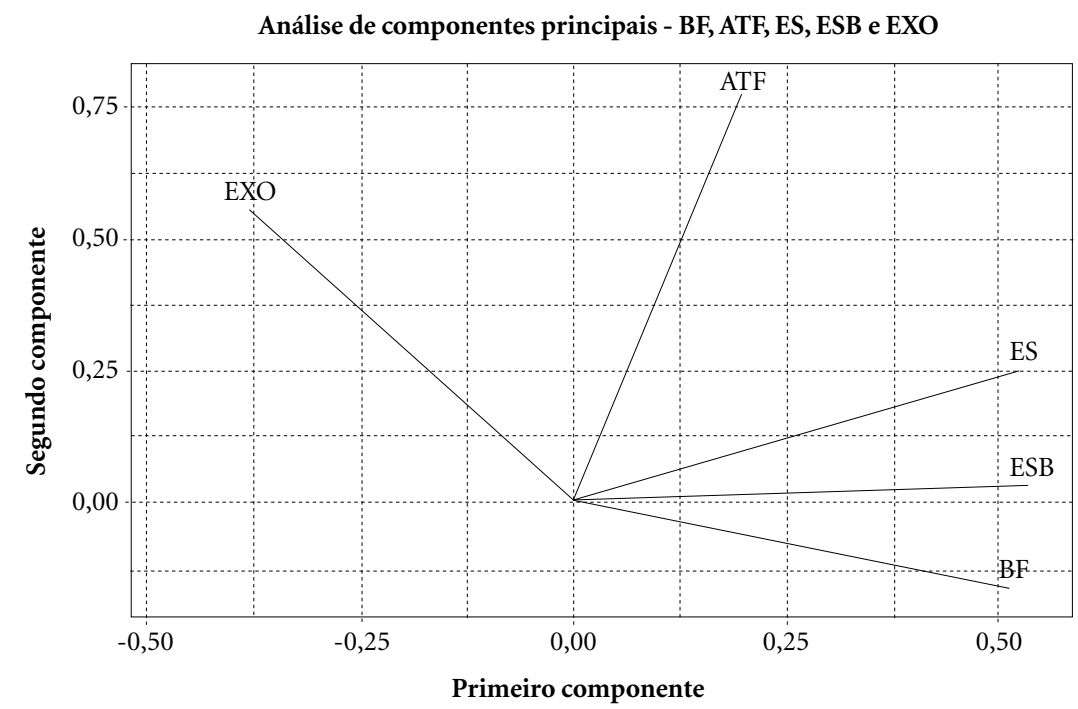

Gráfico 2. Análise multivariada pelo método de componentes principais, apresentando as relações positivas e negativas entre as variáveis BF, ES, ESB, ATF e EXO.

Fonte: Elaborado pelos autores.

vem ser utilizados para subsidiar o planejamento, assim como o monitoramento e avaliação dos indicadores devem ser realizados para qualificar as ações ${ }^{19}$. Verifica-se na literatura alguns traba- $\operatorname{lhos}^{4-6,11,13-15,20,21}$, que monitoram e avaliam procedimentos ambulatoriais na Odontologia apresentados nos sistemas de informações oficiais do Ministério da Saúde; entretanto, trabalhos que 
associaram a implantação das ESBs com o acesso, a realização das ações coletivas e exodontias utilizando dados do SIA-SUS ${ }^{5,13-16}$ são escassos. Até o momento, não foi possível encontrar estudos longitudinais que buscaram analisar o impacto da implantação de Equipes de Saúde Bucal (ESB) sobre a realização de ações coletivas e redução de exodontias no estado do Paraná.

A incorporação do cirurgião-dentista à Equipe de Saúde da Família aconteceu por meio da Portaria 1.444/2000 do Ministério da Saúde ${ }^{22}$, surgindo como uma estratégia para o fortalecimento da Atenção Básica, que se fundamenta no estabelecimento de territórios para o cuidado prioritário, controle das doenças bucais e seu impacto epidemiológico em médio e longo pra$\operatorname{zos}^{23}$. Neste estudo, foi verificado um aumento progressivo da implantação de ESB no Paraná, com um aumento de 47,1\% de 2006 a 2015. Estes dados corroboram com os estudos de Antunes e Narvai ${ }^{21}$, que demonstraram um crescimento expressivo de ESB em todo o Brasil desde 2000. Em outro estudo realizado por Oliveira et al. ${ }^{14}$ no estado do Ceará, os autores também verificaram um aumento, tendo uma grande cobertura populacional pelas ESBs de 77,5\% em 2007. A adesão à Estratégia Saúde da Família (ESF) tem sido priorizada por alguns estados e é almejada, tendo em vista a mudança no modelo de atenção, além da incorporação de ESB estar associada ao aumento de uso do serviço Odontológico no SUS ${ }^{16}$. Além disso, o aumento de ESB nos municípios facilitaria o acesso da população às atividades de promoção, prevenção e educação em saúde, pois um número maior de profissionais atuando na ESF poderia levar à redução da demanda por procedimentos ambulatoriais e especializados individuais ao longo do tempo. Assim, a ESB junto com a equipe multiprofissional trabalhando o cuidado integral em seu território, poderia interferir nos fatores sociais, ambientais, econômicos e emocionais, e facilitar o acesso aos serviços odontológicos, à ações de promoção da saúde geral e bucal. No entanto, apesar do aumento de ESB ocorrido no Paraná, a cobertura pelas ESBs ainda é baixa, demonstrando um grande potencial de expansão a ser desenvolvido no estado.

De acordo com as diretrizes da Política $\mathrm{Na}$ cional de Saúde Bucal ${ }^{19}$, as atividades preventivas e coletivas das ESB possuem de $15 \%$ a $25 \%$ da carga horária de trabalho, que são dedicadas ao diagnóstico do território e planejamento de ações, tendo como foco a família e a comunidade. Essas atividades têm o objetivo de controlar as doenças bucais como a cárie, doença periodontal e câncer bucal, e educar a população para a aquisição de bons hábitos de saúde. Em nosso estudo, verificou-se que as ações coletivas (BF, ES, ATF e EB) vêm aumentando no decorrer dos anos. Estes achados estão de acordo com os trabalhos realizados em Santa Catarina ${ }^{20}$, em municípios do Sul do Brasil ${ }^{10}$ e municípios de todo o Brasil ${ }^{15,16} \mathrm{em}$ diferentes tempos de análise. Almeida e Ferreira ${ }^{24}$ ressaltam que, ao contrário das atividades individuais, as ações em grupos populacionais são expressivas na ESF, rompendo-se com as barreiras do consultório e do modelo tradicional voltado para o individualismo e curativismo. Esses autores também encontraram que a ação coletiva ES foi a mais registrada em Natal em 2006, no estado do Rio Grande do Norte, seguida da ATF. No presente trabalho, as atividades preventivas coletivas mais registradas no SIA-SUS no Paraná, nos anos de 2006 a 2015, foram o BF e a ES. Isto pode ser explicado pelo fato de o Paraná ter um Programa Estadual de Bochecho com Flúor criado em 1980 (Decreto Estadual 3.046/1980), que consiste no fornecimento pelo estado de sachês de 1 grama de Fluoreto de Sódio, que são enviados às prefeituras, para distribuição nas escolas municipais e estaduais. Atualmente, tem sido sugerida a associação dos bochechos à escovação supervisionada, para maior eficácia desta medida preventiva.

Em relação ao indicador EXO, verificou-se neste estudo uma redução de 37,1\% em 9 anos, passando de 43,9 em 2006 para 27,6 em 2015. Da mesma maneira, Peres et al..$^{25}$, verificaram por meio de análise comparativa entre o SB Brasil $2003^{26}$ e o SB Brasil $2010^{27}$, redução no número de dentes perdidos de 0,96 para 0,40 em adolescentes e de 13,5 para 7,4 em adultos. Celeste et al. ${ }^{15}$ também observaram um decréscimo nas tendências das taxas de exodontias em um estudo em municípios de todo o Brasil no período de 1994 a 2007. Por outro lado, alguns estudos demonstraram o aumento da taxa de EXO em municípios com maiores cobertura pela ESB, o que era esperado no início da implantação do programa, tendo em vista as necessidades acumuladas ao longo dos anos ${ }^{10}$. Corrêa e Celeste ${ }^{16}$ encontraram um aumento de $74,1 \%$ nas exodontias, em todos os municípios do país no período de 1999 a 2011, fato este que poderia ser explicado pelo aumento do uso dos serviços odontológicos públicos no Brasil. Além disso, maiores taxas de exodontia foram encontradas em municípios com piores indicadores socioeconômicos, indicando uma alta carga de doença em estágio avançado, para a qual não havia acesso ao tratamento ${ }^{14,20}$.

Em nosso estudo, houve 2 fases de comportamentos distintos: um aumento do indicador 
EXO nos anos de 2006 a 2011, e após esse período, uma redução progressiva até 2015. É possível que nos primeiros anos tenha havido um aumento do acesso e atendimento de uma demanda reprimida com necessidades de procedimentos mais complexos e nos anos subsequentes, a queda no número de exodontias, que poderia ser o reflexo da atuação das ESB, que acumula experiências e vínculo com a população. Não é possível atribuir essas explicações da redução das exodontias somente às variáveis estudadas, já que vários outros fatores poderiam interferir no perfil dos dados apresentados, como o número de profissionais contratados, políticas locais, capacitações realizadas para os profissionais, implementação da rede de atenção à saúde bucal no Paraná ${ }^{28}$, dentre outros.

Considerando que a redução das exodontias de dentes permanentes parece ser um dos impactos mais relevantes da atuação das ESB, ou seja, conseguir interferir no processo saúde-doença de maneira a preservar os dentes, os achados do presente estudo apontaram para um cenário favorável no estado do Paraná. Sabe-se que há um descompasso entre a produção do conhecimento por meio de pesquisas científicas e a sua implementação na prática diária dos serviços de saúde, com um atraso de mais de $10 \operatorname{anos}^{29}$. Assim, é provável que a mudança no processo de trabalho caminhe de acordo com a compreensão dos profissionais, que podem realizar as mudanças necessárias internas (modelos mentais) ou externas (práticas) imediatamente ou mais vagarosamente. Por isso, a construção de séries históricas pode auxiliar na compreensão dos fenômenos que ocorrem na população, já que modula as variáveis ao longo do tempo, podendo revelar as tendências e o lento e natural processo de mudança. Certamente as políticas macro e micro interferem diretamente neste caminhar, e neste aspecto, o estado do Paraná desde 2011 tem buscado fortalecer a Atenção Primária por meio do Programa de Qualificação da Atenção Primária à Saúde (APSUS) ${ }^{30}$, incluindo a saúde bucal. Esta qualificação de toda a equipe multiprofissional no Paraná também pode ter contribuído para os achados deste estudo.

Os resultados deste trabalho apontaram forte correlação positiva entre ESB com os procedimentos coletivos de ES e BF e correlação negativa com exodontia, demonstrando que o aumento do número de cirurgiões-dentistas nas ESB pode ter contribuído para o aumento da quantidade de procedimentos coletivos executados e consequentemente, na redução de exodontias. Esses achados estão de acordo com Palmier et al. ${ }^{5}$ que relatam que a implantação das ESBs contribuiu para os resultados favoráveis dos indicadores de cobertura, procedimentos odontológicos e redução de exodontias nos 52 municípios do Vale do Jequitinhonha. Por outro lado, no estudo de Marques et al. ${ }^{13}$ foram encontradas correlações positivas entre 1a. consulta e exodontia $(r=0,65)$, número de $\mathrm{ESB}$ e primeira consulta $(\mathrm{r}=0,75)$ e ESB e exodontia $(r=0,66)$ no ano de 2012, na Região Metropolitana de Curitiba. Esses autores realizaram o trabalho no mesmo estado do presente estudo, porém, num período de 1 ano, não permitindo uma análise temporal ampla que permitisse verificar mudanças marcantes no comportamento dos indicadores. No estudo de Oliveira et. al. ${ }^{14}$, foi encontrada correlação positiva entre exodontias e ações odontológicas básicas individuais $(r=0,85)$ entre os anos de 2001 a 2007 no estado do Ceará. Esta análise difere do presente estudo, já que a associação não ocorreu com a implantação de equipes de saúde bucal, mas foi relacionada aos procedimentos odontológicos básicos individuais.

É necessário considerar que os dados levantados neste estudo, mesmo tendo sido obtidos de fontes oficiais do Sistema de Informação do Ministério da Saúde, podem ser suscetíveis a possíveis falhas de notificação, pois as orientações dos manuais do SIA-SUS nem sempre são compreendidas e seguidas adequadamente. Os sistemas ainda são fragmentados, o que dificulta o acesso aos dados, havendo a mudança nos códigos e descrição dos procedimentos conforme determina a gestão vigente.

Portanto, algumas inconsistências nos valores dos indicadores entre os anos compreendidos no estudo podem estar presentes, como exemplo, nos valores de EB observados nos anos de 2011 e 2013. Mas, é importante considerar que no ano de 2011 foi publicada a Portaria 2.488, que aprova a Política Nacional de Atenção Básica ${ }^{31}$, estabelecendo a revisão de diretrizes e normas para a organização da Atenção Básica, para a Estratégia Saúde da Família (ESF) e o Programa de Agentes Comunitários de Saúde (PACS). Coincidentemente, foi o ano em que ocorreram os supralançamentos no SIA, o que pode ter refletido em maior realização de EB por parte dos profissionais de saúde, e consequentemente, ocasionado a discrepância em relação aos anos anteriores. Embora houvesse uma queda nos lançamentos EB em 2012, que resultou na redução do indicador deste referido ano, foi perceptível o aumento desse indicador nos anos subsequentes, os quais 
mantiveram-se mais elevados em comparação aos anos anteriores à publicação da Portaria. Desta forma, a Portaria pode ter provocado um marco no incentivo à realização de procedimentos ambulatoriais, sendo um fator positivo não só nos exames bucais como na escovação supervisionada e bochechos fluorados. Outro fator a ser considerado é que o estado do Paraná passou por um processo de reorganização da Atenção Básica, com capacitação dos profissionais de saúde bucal, no período compreendido entre 2011 e 2014 . Foram estabelecidas novas diretrizes básicas da Política Estadual de Saúde Bucal no ano de 2014, reeditadas posteriormente em $2016^{28}$.

O presente estudo foi realizado com dados oriundos dos 399 municípios do Paraná, apresentando informações regionais relevantes, que podem apresentar resultados das políticas públicas adotadas no estado nos últimos anos, além de auxiliar no planejamento de ações futuras por gestores e pesquisadores. Outros estudos são necessários para melhor compreender os fenômenos estudados neste trabalho, complementar com variáveis socioeconômicas, condição de saúde bucal, processo de trabalho dos profissionais, dentre outras, e poder avaliar fatores associados aos impactos da implantação das ESB no Paraná ou em outras partes do Brasil.

\section{Conclusão}

Assim, conclui-se que a implantação das Equipes de Saúde Bucal pode ter contribuído para o aumento das ações coletivas e redução do número de exodontias no estado do Paraná. A implantação das ESB no estado do Paraná é um processo em expansão, com potencial de crescimento.

\section{Colaboradores}

PM Siqueira e JE Umeda trabalharam na coleta de dados, estatística e redação final. RSS Terada e M Fujimaki trabalharam na concepção da pesquisa e determinação da metodologia a ser aplicada. JS Leite, AF Giozet e M Paludetto Junior realizaram a busca bibliográfica para redação da introdução e discussão. CCB Lima auxiliou na análise estatística e revisão do texto.

\section{Agradecimentos}

O presente trabalho foi realizado com apoio da Coordenação de Aperfeiçoamento de Pessoal de Nível Superior - Brasil (CAPES) - Código de Financiamento 001 e Programa Nacional de Cooperação Acadêmica (PROCAD) 2013/CAPES. 


\section{Referências}

1. Souza AN. Monitoramento e avaliação na Atenção Básica no Brasil: a experiência recente e desafios para a consolidação. Saude Debate 2018; 42(1):289-301.

2. Brasil. Ministério da Saúde (MS). Manual técnico operacional SIA/SUS - Sistema de Informações. Brasília: MS; 2010.

3. Brasil. Ministério da Saúde (MS).e-SUS Atenção Básica: Manual do Aplicativo ACS - Agente Comunitário de Saúde. Brasília: MS; 2015.

4. Barros SG, Chaves SCL. A utilização do Sistema de Informações Ambulatoriais (SIA-SUS) como instrumento para caracterização das ações de saúde bucal. Epidemiol Serv Saude 2003; 12(1):41-51.

5. Palmier AC, Andrade DA, Campos ACV, Abreu MHNG, Ferreira EF. Indicadores socioeconômicos e serviços odontológicos em uma região brasileira desfavorecida. Rev Panam Salud Publica 2012; 32(1):2229.

6. Pereira CRS, Patrício AAR, Araújo FAC, Lucena EES, Lima KC, Roncalli AG. Impacto da Estratégia Saúde da Família com equipe de saúde bucal sobre a utilização de serviços odontológicos. Cad Saude Publica 2009; 25(5):985-996.

7. Paula JS, Ambrosano GMB, Mialhe FL. The impact of social determinants on schoolchildren's oral health in Brazil. Braz Oral Res 2015; 29(1):1-9.

8. Bueno VLRC, Cordoni Júnior L, Mesas AE. Desenvolvimento de indicadores para avaliação de serviço público de odontologia. Cien Saude Colet 2011; 16(7):3069-3082.

9. Pucca Jr GA, Gabriel M, Araujo ME, Almeida FCS. Ten Years of a National Oral Health Policy in Brazil: Innovation, Boldness, and Numerous Challenges. $J$ Dent Res 2015; 94(10):1333-1337.

10. Fischer TK, Peres KG, Kupek E, Peres MA. Indicadores de atenção básica em saúde bucal: associação com as condições socioeconômicas, provisão de serviços, fluoretação de águas e a estratégia de saúde da família no Sul do Brasil. Rev Bras Epidemiol 2010; 13(1):126-138.

11. Teixeira ND, Facchini LA, Castilhos ED. Avaliação da evolução da demanda de saúde bucal através do uso de sistemas de informação em saúde. Rev Enferm Saude 2011; 1(1):50-59.

12. Baldani MH, Almeida ES, Antunes JLF. Eqüidade e provisão de serviços públicos odontológicos no estado do Paraná. Rev Saude Publica 2009; 43(3):446-454

13. Marques AB, Oneda G, Buffon MCM, Ditterich RG. Sistemas de informação como ferramenta de monitoramento das ações de saúde bucal na Estratégia Saúde da Família da região metropolitana de Curitiba-PR. Rev Bras Pesq Saude 2014; 16(1):82-89.

14. Oliveira PMC, Menezes LMB, Santrain MVL, Almeida PC, Almeida MEL. Indicadores de saúde bucal da atenção básica no estado do Ceará: Análise crítica. Cad ESP 2011; 5(1):29-36.

15. Celeste RK, Vital JF, Junger WL, Reichenheim ME. Séries de procedimentos odontológicos realizadas nos serviços públicos brasileiros, 1994-2007. Cien Saude Colet 2011; 16(11):4523-4532.
16. Corrêa GT, Celeste RK. Associação entre a cobertura de equipes de saúde bucal na saúde da família e o aumento na produção ambulatorial dos municípios brasileiros, 1999 e 2011. Cad Saude Publica 2015; 31(12):2588-2598.

17. Instituto Brasileiro de Geografia e Estatística (IBGE). Projeção da população do Paraná de 2000-2030 [Internet]. [acessado 2016 out 20]. Disponível em: http:// www.ibge.gov.br.

18. Conselho Nacional de Secretários de Saúde (CONASS). Indicadores: Cobertura de Equipes de Saúde da Família e de Saúde Bucal [Internet]. [acessado 2016 out 26]. Disponível em: http://www.conass.org.br/ guiainformacao/notas_tecnicas/NT6-Cobertura-ESF-e-ESB.pdf.

19. Brasil. Ministério da Saúde (MS). Coordenação Geral de Saúde Bucal. Diretrizes da Política Nacional de Saúde Bucal. Brasília: MS; 2004.

20. Fernandes LS, Peres MA. Associação entre atenção básica em saúde bucal e indicadores socioeconômicos municipais. Rev Saude Publica 2005; 39(6):930-936.

21. Antunes JFL, Narvai PC. Políticas de saúde bucal no Brasil e seu impacto sobre as desigualdades em saúde. Rev Saude Publica 2010; 44(2):360-365.

22. Brasil. Portaria $n^{\circ} 1.444$, de 28 de dezembro de 2000. Estabelece incentivo financeiro para a reorganização da atenção à saúde bucal prestada nos municípios por meio do Programa de Saúde da Família. Diário Oficial da União 2000; $28 \mathrm{dez}$.

23. Junqueira SR, Pannuti CM, Rode SM. Oral Health in Brazil-Part I: Public Oral Health Policies. Braz Oral Res 2008; 22(esp. 1):8-17.

24. Almeida GCM, Ferreira MAF. Saúde bucal no contexto do Programa Saúde da Família: práticas de prevenção orientadas ao indivíduo e ao coletivo. Cad Saude Publica 2008; 24(9):2131-2140.

25. Peres MA, Barbato PR, Reis SCGB, Freitas CHSDM, Antunes JLF. Tooth loss in Brazil: Analysis of the 2010 Brazilian oral health survey. Rev Saude Publica 2013; 47(3):78-89.

26. Brasil. Ministério da Saúde (MS). Secretaria de Atenção à Saúde.Departamento de Atenção Básica.Coordenação Nacional de Saúde Bucal. Projeto SB Brasil 2003. Condições de saúde bucal da população brasileira 2002-2003. Resultados principais. Brasília: MS; 2004.

27. Brasil. Ministério da Saúde (MS). Secretaria de Atenção à Saúde. Secretaria de Vigilância à Saúde. SB Brasil 2010: Pesquisa Nacional de Saúde Bucal: resultados principais. Brasília: MS; 2012.

28. Secretaria de Estado da Saúde do Paraná. Superintendência de Assistência à Saúde. Linha guia rede de saúde bucal. Curitiba: SESA; 2016.

29. Balas EA, Boren SA. Managing Clinical Knowledge for Health Care Improvement. Yearb Med Inform 2000; 1:65-70.

30. Secretaria de Estado da Saúde do Paraná. A Tutoria na Atenção Primária à Saúde. Curitiba: SESA; 2018. 
31. Brasil. Portaria $\mathrm{n}^{\circ} 2.488$, de 21 de outubro de 2011. Aprova a Política Nacional de Atenção Básica, estabelecendo a revisão de diretrizes e normas para a organização da Atenção Básica, para a Estratégia Saúde da Família (ESF) e o Programa de Agentes Comunitários de Saúde (PACS). Diário Oficial da União 2011; 21 out.

Artigo apresentado em 25/09/2018

Aprovado em 06/04/2020

Versão final apresentada em 08/04/2020

Editores-chefes: Romeu Gomes, Antônio Augusto Moura da Silva 